\title{
Flipped Reposition Laminoplasty for Excision of Intradural Extramedullary Tumors in the Thoracolumbar Spine: A Case Series of 14 Patients
}

\author{
Ramesh Kumar, Ijack Debbarma, Tankeshwar Boruah, Atul Sareen, \\ Mohit Kumar Patralekh, Ashish Dagar, Shaffaf Abdul Kareem \\ Central Institute of Orthopaedics, Vardhman Mahavir Medical College and Safdarjung Hospital, New Delhi, India
}

Study Design: A retrospective study was done to assess the outcome of the new technique of flipped reposition laminoplasty for excision of intradural extramedullary (IDEM) spinal tumors of the thoracolumbar region.

Purpose: To describe flipped reposition laminoplasty technique and evaluate its outcomes.

Overview of Literature: Laminectomy has been the conventional approach for the surgical excision of IDEM spinal tumors, but it has potential postoperative complications. Laminoplasty maintains the posterior arch of the spine and avoids complications seen in Laminectomy, such as instability, epidural scarring, and kyphotic deformity.

Methods: Fourteen patients (nine females and five males) diagnosed with IDEM tumors of the thoracolumbar region operated between 2016 and 2018 were included in this study. Pathologically, five cases were schwannomas; four cases were meningiomas; two cases were ependymomas; and one case each was lymphoma, neurofibroma, and teratoma. All patients had their neurological deficits documented using the American Spinal Injury Association (ASIA) impairment scale. After completion of all preanesthetic formalities, the patients were operated upon by a single surgeon using the flipped reposition laminoplasty technique. Follow-up was done at 1, 3, 6, and 12 months post operation and yearly thereafter.

Results: The mean age of the patients was 35.28 years (14-65 years), and the mean follow-up duration was 17 months (6-26 months). Two patients were assessed with ASIA grade A neurology, one patient improved to ASIA grade B, whereas the other did not improve. Two patients improved from ASIA grade $B$ to $A S I A$ grade $D$, and seven patients with $A S I A$ grades $C$ and $D$ improved to $A S I A$ grade E. Fusion at the osteotomy site was seen in $92.85 \%$ (13 out of 14) cases on one side within 6 months post operation. Fusion was seen in all the cases within 1 year post operation.

Conclusions: Flipped reposition laminoplasty is an excellent technique providing adequate exposure and additional stability postoperatively.

Keywords: Laminectomy; Flipped reposition laminoplasty; Intradural extramedullary; American Spinal Injury Association impairment scale

Received Jan 29, 2019; Revised Jul 14, 2019; Accepted Aug 6, 2019

Corresponding author: Ijack Debbarma

Central Institute of Orthopaedics, VMMC and Safdarjung Hospital, New Delhi, 110029, India

Tel: +91-8126685367, Fax: +91-011-2673-0299, E-mail: ijack1987@gmail.com 


\section{Introduction}

Spinal tumors comprise $15 \%$ of all central nervous system tumors. Their annual incidence is $2-10$ per 100,000 people [1]. Intradural extramedullary (IDEM) spinal tumors are rare but are the most commonly observed intradural spinal tumors, comprising over $60 \%$ of tumors found within the spinal canal [2].

Various surgical techniques have been described in the literature for IDEM tumor excision. Laminectomy has been the conventional workhorse approach, but it has several potential complications, including hematoma invasion, postoperative spinal instability, epidural fibrosis, kyphotic deformity, excessive blood loss, progressive myelopathy, persistent back pain, and prolonged hospital stay [3-5]. Considering that IDEM tumors can recur [1], revision surgery is also an issue with laminectomy as the dura is left without bony cover, making exposure during the repeat surgery a challenge.

Laminoplasty is widely used in thoracic and lumbar spinal surgery for intradural tumor excision. It provides wide exposure; retains the posterior arch of the spine; and prevents postoperative instability, epidural scarring, back pain, and kyphotic deformity [6].

Many types of laminoplasties have been described in the literature, such as T-saw, Y, transverse placement, restorative, inverse, and en bloc laminoplasties; each type has unique advantages and disadvantages [7-11].

Supraspinous and interspinous ligaments are important parts of the posterior ligamentous complex and act as dynamic stabilizers of the spine. A porcine study has reported more adjacent segment instability when the supraspinous and interspinous ligaments are not anchored [12].

In this study, we introduce our surgical technique of "flipped reposition laminoplasty," a modification of the standard laminoplasty technique, for the excision of IDEM tumors of the thoracolumbar spine. In this technique, posterior laminoplasty flap is raised, leaving the cephalad part of the supraspinous and interspinous ligaments intact. This technique may provide additional stability postoperatively, and the aim of this study is to assess the outcome of this technique.

\section{Materials and Methods}

\section{Participants}

A hospital-based retrospective study with prospective follow-up data collection was conducted with a study population of 14 patients operated between 2016 and 2018 with a primary diagnosis of IDEM tumors of the thoracolumbar spine. Patients having IDEM tumors with bony involvement were excluded from this study. All study participants were admitted through outpatients or the emergency department. Diagnosis was made based on clinical and radiological examination. The patients were evaluated preoperatively with routine blood investigations, plain X-rays, computed tomography (CT) scans, and magnetic resonance imaging (MRI). Neurological deficits were documented using the American Spinal injury Association (ASIA) impairment scale [13]. Informed written consent for the surgical procedure was taken from all patients. Preanesthetic fitness was ascertained according to our hospital protocol. It was a retrospective with prospective follow-up study design, so ethical approval was not required.

\section{Surgical technique}

After general anesthesia, the patient was positioned prone. A standard posterior midline approach was used. Paraspinal muscles were dissected subperiosteally, leaving the supra- and interspinous ligaments and the facet joint capsule intact. When required, pedicle screws were inserted at desired levels using freehand technique and confirmed by c-arm. The lamina of the desired level to be decompressed is identified and confirmed by c-arm. The graft bed was prepared on both sides of the lamina before the cuts were made. Osteotomy was performed on both sides of the lamina just medial to the facet joint with the help of an ultrasonic bone scalpel (Fig. 1). An inferior cut was made through the superior half of the spinous process of the inferior vertebra. The osseo-ligamentous flap thus created was gradually elevated en bloc, starting distally using a curette and a McDonald dura retractor, leaving the superior end intact (Fig. 2). The osseo-ligamentous flap was then flipped superolaterally, hinging over the superior interspinous ligament to expose the spinal canal and its contents. Hemostasis was maintained using bipolar cautery and platelet aggregating hemostat. The dural sheath was 


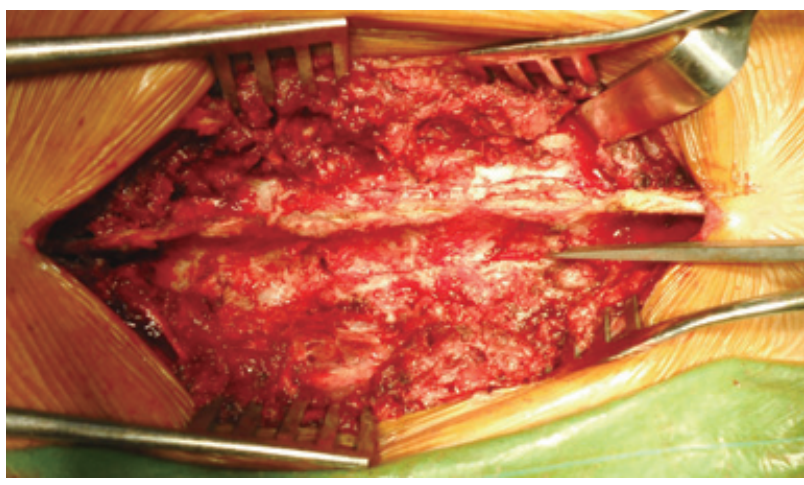

Fig. 1. Laminoplasty osteotomy made medial to facet joint and pars.

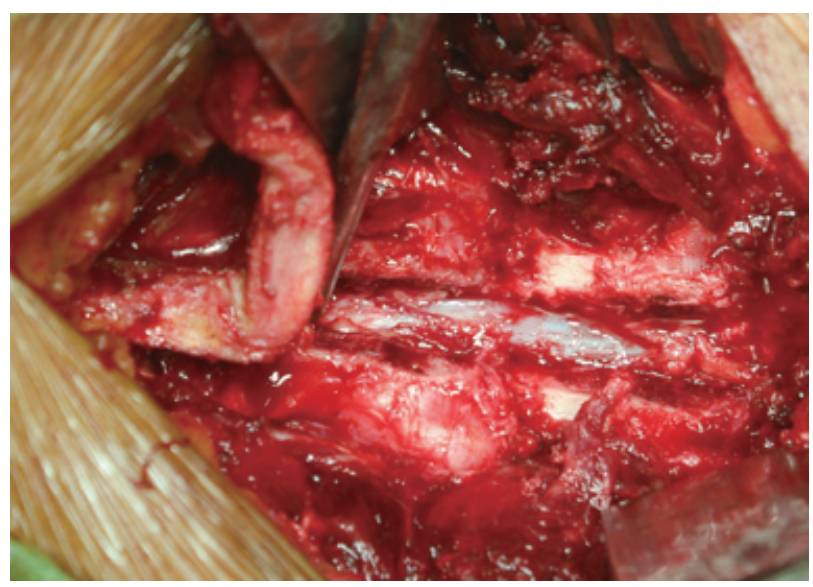

Fig. 2. Laminoplasty flap flipped keeping cranial end intact.

incised carefully along the tumor bulge in the midline. The tumor mass was excised en bloc by blunt dissection using a Penfield dissector (Fig. 3). The dural sheath was sutured with 6-0 non-absorbable suture. Instrumentation of the spine was then completed using rods and cross connectors if indicated.

Any sharp, bony prominences along the flap margins were smoothened. The posterior arch was then recreated by repositioning the flap that was secured using Ethibond 3-0 suture through the flap and remaining inferior spinous process (Fig. 4). A mixture of autogenous and artificial bone graft was then placed over the previously prepared graft bed if indication for fusion was present. We performed fusion with pedicle screw instrumentation if it was necessary to cut more than two laminae to achieve adequate exposure for tumor removal or if more than half of the facet joint was excised to gain sufficient lateral exposure. The incision was closed in layers with a drain. We used titanium miniplates to secure the laminoplasty for motion-preserving cases in patients who could afford it. In other cases, we used ethibond for this purpose (for

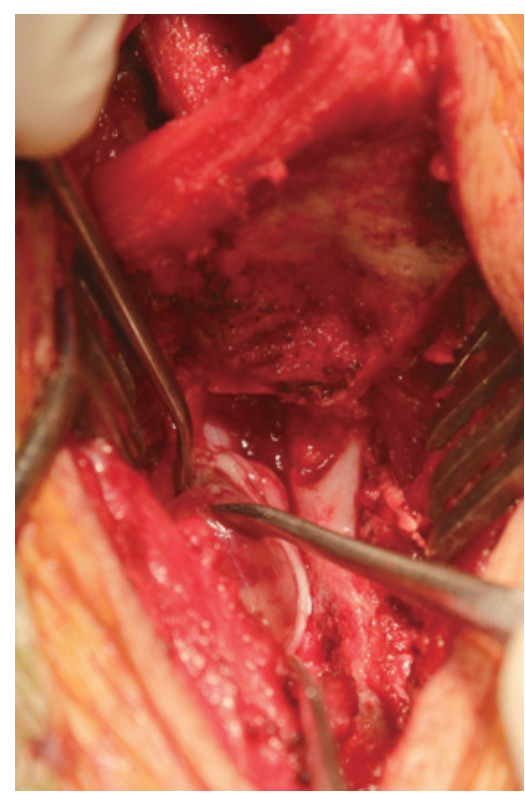

Fig. 3. Tumor removal.

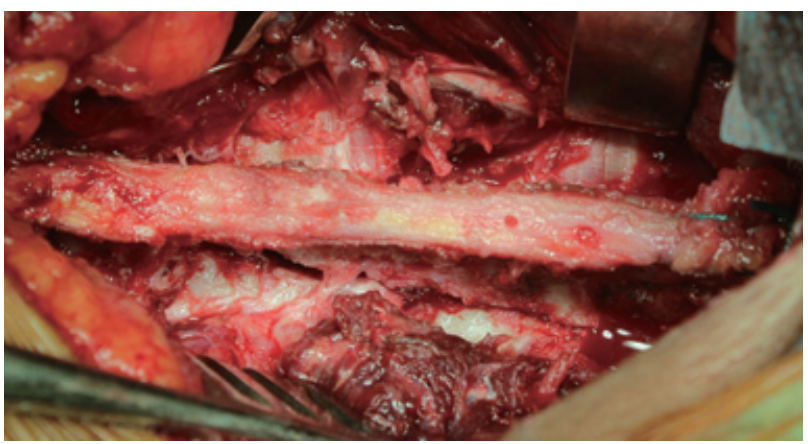

Fig. 4. Final reconstruction of laminoplasty flap.

cases requiring motion preservation and cases requiring fusion). Ethibond suture or miniplates provide additional security against sinking in of the laminoplasty flap.

The tumor size was measured and sent for histopathological examination. Blood loss during the surgery was measured by gauze visual analog technique [14], and the duration of the surgery, skin to skin, was noted.

During the postoperative period, a suitable Brace was prescribed and mobilization was started after suture removal in 2 weeks.

\section{Follow-up}

Patients were followed up at 1, 3, 6, and 12 months and yearly thereafter and assessed clinically and radiologically. The clinical outcome was assessed by neurological examination according to the ASIA impairment scale. Radiological assessment was done using plain flexion-extension 
radiograph to assess any postoperative instability. Measuring restoration of the size of the spinal canal and fusion of the osteotomy site was based on CT scan.

\section{Results}

The total study population consisted of 14 patients ( 9 [64.28\%] females and 5 [35.71\%] males) with a mean age of 35.28 years (range, $14-65$ years). The minimum followup duration was 6 months while the maximum was 26 months (mean, 17 months).

Pathologically, five cases were schwannomas, four cases were meningiomas, two cases were ependymomas, and one case each was neurofibroma, teratoma, and lymphoma. The site of the lesion was the proximal thoracic region (D1-D4) in five cases, the thoracolumbar junction (D10-L2) in five cases, and the mid-dorsal region (D5D9) in three cases. The least common site was the lumbar spine (L3-L5), having only one case. At the preoperative neurological examination, six, two, two, and two patients were ASIA grades D, A, B, and C, respectively, whereas two patients had intact neurology.

Out of the14 patients, one-level, two-level, three-level, and four-level laminoplasties were done in two, six, five, and one cases, respectively. Motion-preserving surgery was done in two cases and fusion was performed in the remaining 12. Ethibond sutures were used in one motionpreserving and 12 fusion cases. Titanium miniplates were used in only one case of motion-preserving surgery. The mean operative time was 2.42 hours (range, 2-3 hours), with an intraoperative mean blood loss of $303.57 \mathrm{~mL}$ (range, 250-450 mL). In one case, a nerve root was attached to the mass and had to be sacrificed for excision intraoperatively.

Two patients developed neurological deterioration in the immediate postoperative period that subsequently improved within 8 weeks. No other complications were seen during the follow-up period.

Out of the two patients with ASIA grade A neurology, one patient improved to ASIA grade B, whereas the other
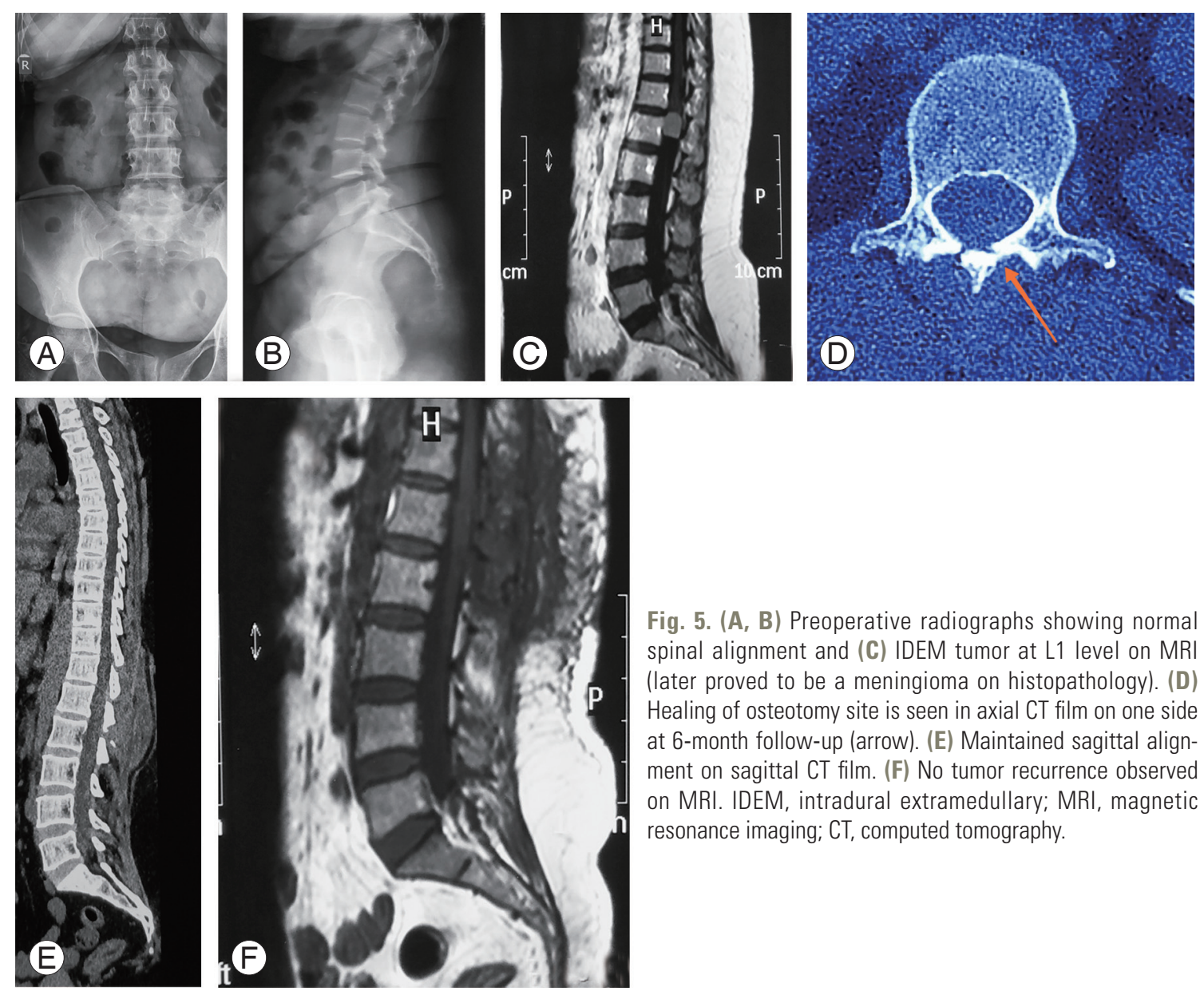

Fig. 5. (A, B) Preoperative radiographs showing normal spinal alignment and (C) IDEM tumor at L1 level on MRI (later proved to be a meningioma on histopathology). (D) Healing of osteotomy site is seen in axial CT film on one side at 6-month follow-up (arrow). (E) Maintained sagittal alignment on sagittal CT film. (F) No tumor recurrence observed on MRI. IDEM, intradural extramedullary; MRI, magnetic resonance imaging; CT, computed tomography. 

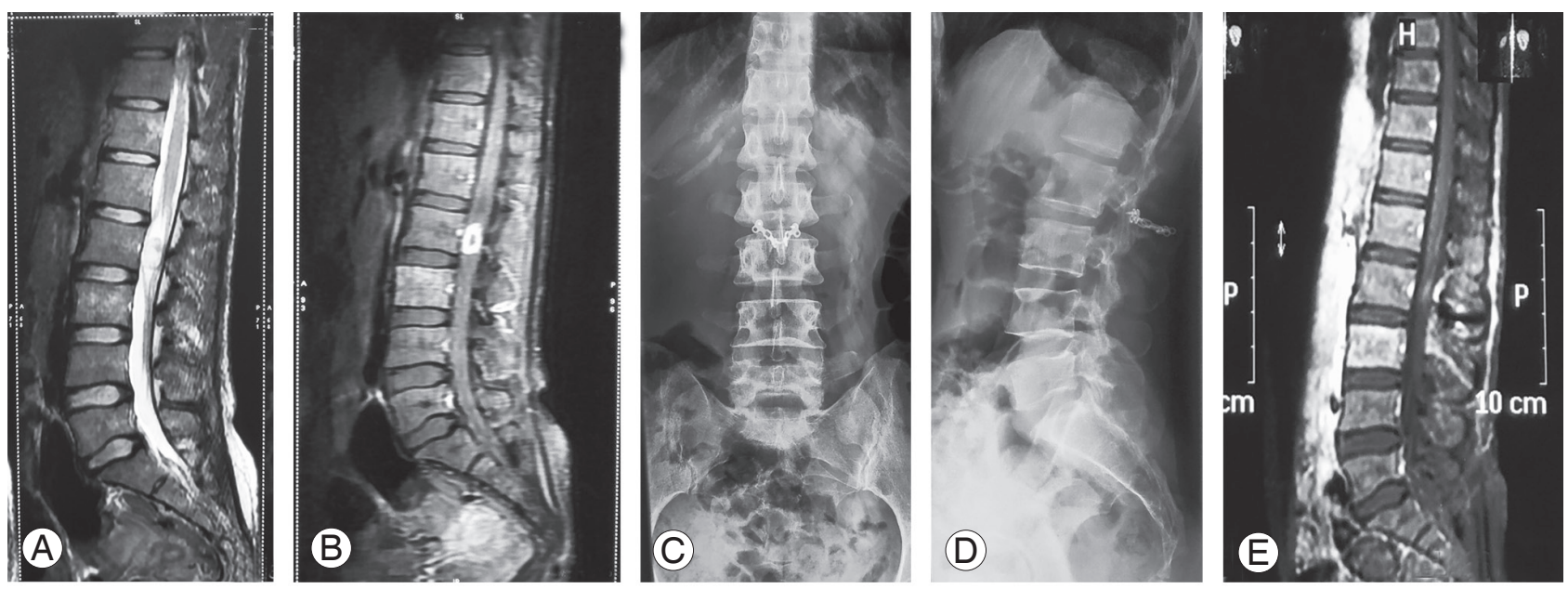

Fig. 6. A case with IDEM tumor at the L2 level. Laminoplasty flap was secured with titanium miniplates. (A, B) Preoperative MRI showing IDEM tumor-hyperintensities on T2W enhancing on T1W postcontrast (tumor proved to be neurofibroma on histopathology). (C, D) Postoperative radiographs demonstrating normal alignment and titanium miniplates in position. (E) MRI showing no tumor. IDEM, intradural extramedullary; MRI, magnetic resonance imaging.

did not improve. Two patients improved from ASIA grade $B$ to ASIA grade D and seven patients improved from ASIA grade $\mathrm{C}$ and $\mathrm{D}$ to grade $\mathrm{E}$.

Fusion of the osteotomy site was seen in $92.85 \%$ (13 out of 14) cases on one side within 6 months post operation, and complete fusion was seen in all the cases within 1 year of follow-up. Spinal alignment and stability was maintained in all cases on follow-up. Fig. 5 illustrates a case with motion preservation in which the laminoplasty flap was secured by Ethibond sutures. Preoperative radiographs show normal spinal alignment (Fig. 5A, B) and the IDEM tumor at L1 level (Fig. 5C); the healing of the osteotomy site is seen in axial CT film on one side at 6-month follow-up (Fig. 5D), along with maintained sagittal alignment visible on the sagittal CT film (Fig. 5E). No tumor recurrence was seen on MRI (Fig. 5F).

Fig. 6 illustrates another case in which the laminoplasty flap was secured with titanium miniplates. The preoperative MRI showed IDEM tumor-hyperintensities on T2W (Fig. 6A), enhancing on T1W postcontrast (Fig. 6B). The postoperative radiographs demonstrated normal alignment and the titanium miniplates in position (Fig. 6C, D). The MRI showing complete excision is seen in (Fig. 6E). No change in alignment was observed on follow-up, as illustrated in Fig. 7.

Recurrence of the tumor was not observed in any of the cases. No spinal instability, kyphotic deformity, surgical site infection, or persistent back pain was observed on follow-up. The results are summarized in Tables 1 and 2.
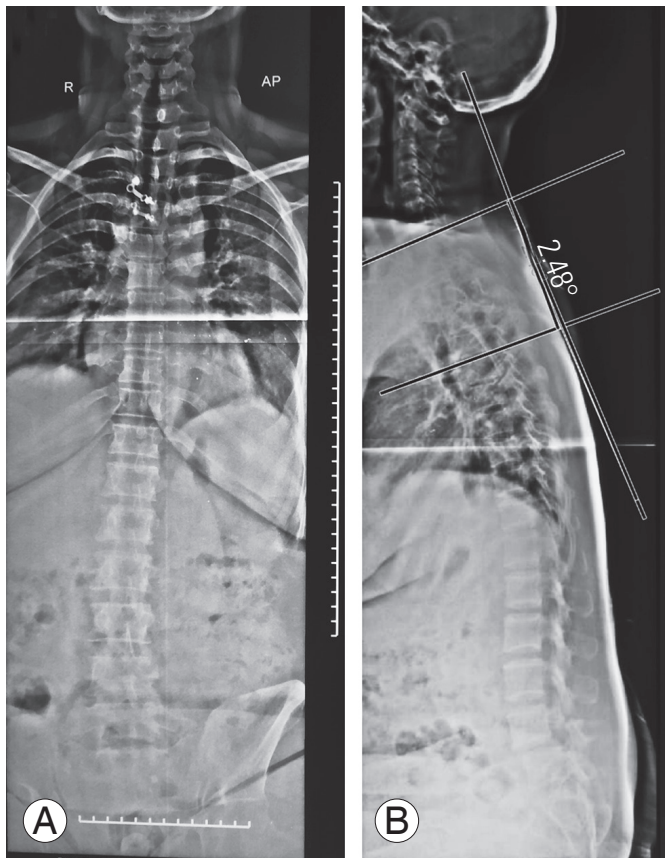

Fig. 7. (A, B) Follow-up orthogonal whole spine radiograph of a case where motion-preserving laminoplasty was done for intradural extramedullary tumor removal and fixed with titanium miniplates at two levels (D3 and D4) for illustration showing preserved coronal and sagittal alignment. Sagittal proximal thoracic cobbs (D1-D5) measures 2.48 ${ }^{\circ}$. AP, anterior-posterior.

\section{Discussion}

Conventional laminectomy provides adequate exposure for most of the IDEM lesions but can lead to several complications, such as postoperative spinal instability, ky- 
Table 1. Summary of results of all cases

\begin{tabular}{|c|c|}
\hline Characteristic & Value \\
\hline Total no. of patients & 14 \\
\hline Age (yr) & $35.28(14-65)$ \\
\hline Sex (male:female) & $5: 9$ \\
\hline \multicolumn{2}{|l|}{ Pathology of tumor } \\
\hline Schwannoma & 5 \\
\hline Meningioma & 4 \\
\hline Ependymoma & 3 \\
\hline Neurofibroma & 1 \\
\hline Teratoma & 1 \\
\hline Lymphoma & 1 \\
\hline \multicolumn{2}{|l|}{ Levels } \\
\hline Proximal thoracic & 5 \\
\hline Mid-thoracic & 3 \\
\hline Thoraco-lumbar & 5 \\
\hline Lumbar & 1 \\
\hline Fusion & 12 \\
\hline Motion preservation & 2 \\
\hline \multicolumn{2}{|c|}{ Laminoplasty flap fixation technique } \\
\hline Ethibond suture & 13 \\
\hline Mini plate & 1 \\
\hline \multicolumn{2}{|c|}{ Neurological status (AIS grade) } \\
\hline \multicolumn{2}{|l|}{ Preoperative } \\
\hline A & 2 \\
\hline B & 2 \\
\hline C & 2 \\
\hline D & 6 \\
\hline Intact & 2 \\
\hline \multicolumn{2}{|l|}{ Postoperative } \\
\hline A & 1 \\
\hline B & 1 \\
\hline C & 0 \\
\hline D & 2 \\
\hline E & 10 \\
\hline
\end{tabular}

Values are presented as number or mean (range). AIS, American Spinal Injury Association impairment scale.

photic deformity, epidural scarring, persistent back pain, and difficulty in revision surgery [3-5]. To reduce the incidence of these complications, laminoplasty has been recommended in various studies [6-11].

The laminoplasty technique provides adequate exposure to the spinal canal while maintaining the posterior arch of the spine and prevents postoperative instability, epidural adhesion, and kyphotic deformity [15]. Considering that IDEM can recur, laminoplasty makes revision surgery much easier, as it is safer to expose the dural sheath after this type of surgery than after traditional laminectomy. Hida et al. [10] reported the results of a more than 2-year follow-up of transverse placement laminoplasty. The report demonstrated that sagittal alignment was better preserved by laminoplasty than by laminectomy [10].

Several types of laminoplasty fixation devices for the thoracolumbar area have been reported, including titanium mini plates and translaminar screws $[10,16]$.

Recapping T-saw laminoplasty as proposed by Miyakoshi et al. [7] for thoracic calcified meningiomas involves cutting from pedicle to transverse process. It can provide extremely wide exposure of the spinal canal. One possible complication of recapping T-saw laminoplasty is neural injury when the T-saw passes beneath the lamina [11]. However, further studies with long-term follow-up are needed to confirm whether late complications like spinal instability occur.

Keeping these factors in mind we adapted our technique for IDEM tumor excision in the thoracolumbar region. We used an ultrasonic bone scalpel instead of a T-saw, which minimizes bone loss and eliminates the risk of neural injury. Performing the osteotomy cuts medial to the facet joint helps in maintaining the spinal stability in contrast to T-saw recapping laminoplasty. Further, leaving the superior end of the flap intact provides additional stability. Using our technique, we achieved adequate decompression of the spinal cord and were also able to replace the flipped structures to their exact anatomical position. The addition of posterolateral fusion with pedicle screws and bone graft provides three-column fixations and helps in fusion of the segment, allowing early mobilization of the patient. During the follow-up period, we found fusion of the osteotomy site in 11 patients within 6 months and in all patients within 1 year.

The hemilaminectomy approach has several advantages over conventional laminectomy $[17,18]$, but it also has several disadvantages, such as limited access and difficulty in dural repair. It can also be more difficult to remove dumbbell tumors (Eden types 2 and 3), malignant lymphomas, and bleeding tumors spreading to both sides and midline tumors $[19,20]$.

Good neurological recovery was observed during the postoperative period as seven patients improved to grade $\mathrm{E}$ and two patients to grade D. One patient developed 


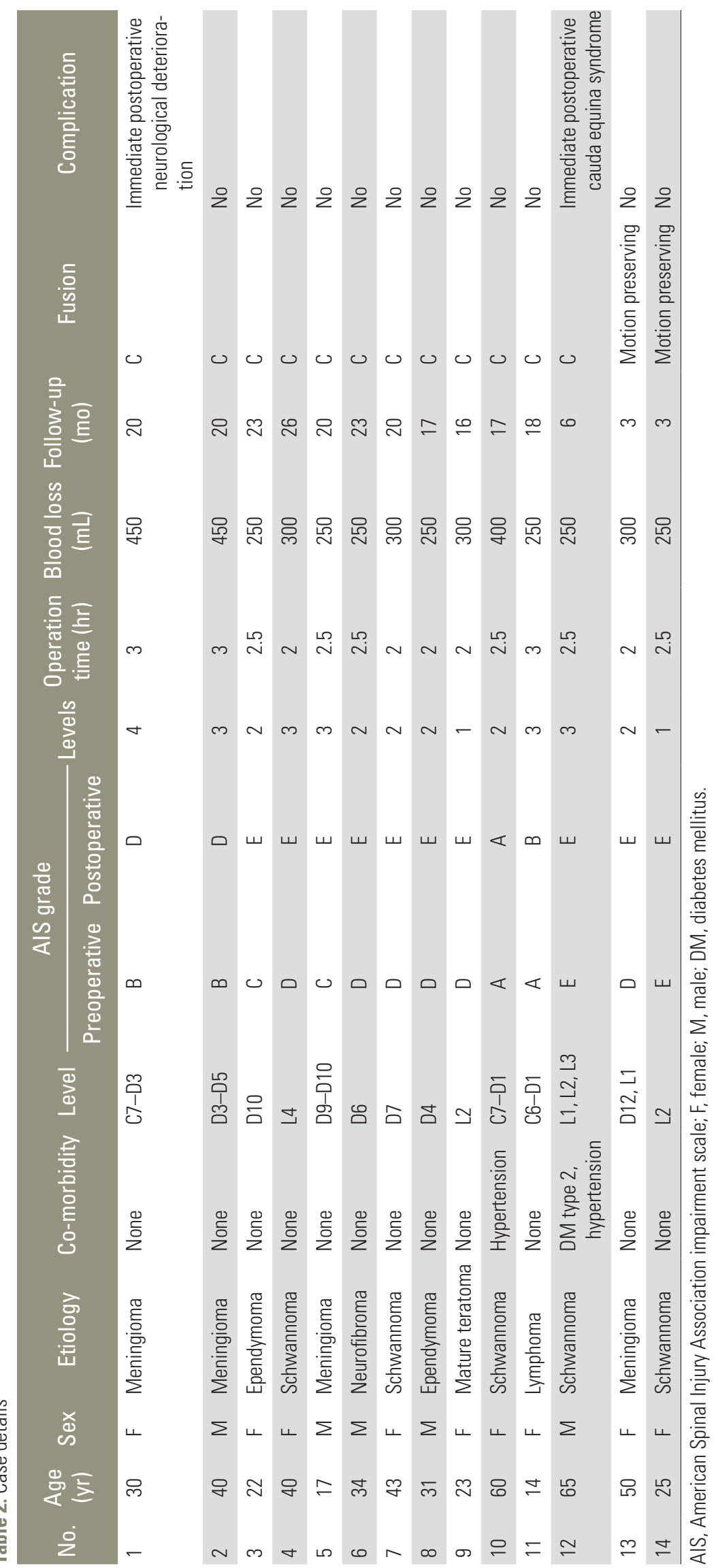


neurological deterioration and one patient developed cauda equina syndrome in the immediate postoperative period that improved on follow-up within 8 weeks. No spinal instability, persistent back pain, or kyphotic deformity was observed in our study group. Tumor recurrence was not observed in any case. Repositioning flip laminoplasty with fusion (i.e., without motion preservation) uses sutures only to secure the laminoplasty flap to the pedicle screws and rods and for securing the caudal spinous process cut. It allows more surface area and raw bone for bone grafting, thereby promoting posterior fusion, as compared with laminectomy or hemilaminectomy. Repositioning flip laminoplasty with motion preservation uses plates to secure the laminoplasty. Metacarpal plates or laminoplasty plates may be used after suitable contouring. Titanium plates are used as they are MRI compatible. Ethibond sutures may also be used alone to secure the laminoplasty flap to adjacent paraspinal muscles to prevent sinking in, and for securing the caudal spinous process cut. Oblique laminar cuts prevent sinking of the flap. This technique preserves the posterior tension band (posterior ligamentous complex), preventing postlaminectomy instability and kyphosis. Thus, it almost restores the original anatomy and biomechanics. For both types of cases, we speculate that our technique of preserving the laminae may also reduce problems like hematoma invasion and post-laminectomy membrane formation, which occur due to the lack of posterior bony cover over the dura. It allows canal expansion and also allows relatively safe exposure in future surgery, if necessary.

The limitations of this study were the small sample size of 14 patients, relatively short follow-up time, and no randomization of the samples.

\section{Conclusions}

Flipped reposition laminoplasty is an excellent technique for the excision of IDEM tumors of the thoracolumbar spine. The technique provides adequate exposure and additional stability postoperatively.

\section{Conflict of Interest}

No potential conflict of interest relevant to this article was reported.

\section{References}

1. Arnautovic K, Arnautovic A. Extramedullary intradural spinal tumors: a review of modern diagnostic and treatment options and a report of a series. Bosn J Basic Med Sci 2009;9 Suppl 1:40-5.

2. Aghayev K, Vrionis F, Chamberlain MC. Adult intradural primary spinal cord tumors. J Natl Compr Canc Netw 2011;9:434-47.

3. Iida Y, Kataoka O, Sho T, et al. Postoperative lumbar spinal instability occurring or progressing secondary to laminectomy. Spine (Phila Pa 1976) 1990;15:11869.

4. Mayfield FH. Complications of laminectomy. Clin Neurosurg 1976;23:435-9.

5. Yasuoka S, Peterson HA, MacCarty CS. Incidence of spinal column deformity after multilevel laminectomy in children and adults. J Neurosurg 1982;57:4415.

6. Liu XY, Zheng YP, Li JM. Laminoplasty for the treatment of extramedullary intradural tumors in the thoracic and lumbar spine: greater than two-year followup. Orthop Surg 2009;1:275-9.

7. Miyakoshi N, Hongo M, Kasukawa Y, Shimada Y. Enbloc resection of thoracic calcified meningioma with inner dural layer in recapping T-saw laminoplasty: a case report. BMC Surg 2015;15:82.

8. Adachi K, Futami T, Ebihara A, et al. Spinal canal enlargement procedure by restorative laminoplasty for the treatment of lumbar canal stenosis. Spine J 2003;3:471-8.

9. Hara M, Takayasu M, Takagi T, Yoshida J. En bloc laminoplasty performed with threadwire saw. Neurosurgery 2001;48:235-9.

10. Hida S, Naito M, Arimizu J, Morishita Y, Nakamura A. The transverse placement laminoplasty using titanium miniplates for the reconstruction of the laminae in thoracic and lumbar lesion. Eur Spine J 2006;15:1292-7.

11. Kawahara N, Tomita K, Shinya Y, et al. Recapping Tsaw laminoplasty for spinal cord tumors. Spine (Phila Pa 1976) 1999;24:1363-70.

12. Chen LH, Lai PL, Tai CL, Niu CC, Fu TS, Chen WJ. The effect of interspinous ligament integrity on adjacent segment instability after lumbar instrumentation and laminectomy: an experimental study in porcine model. Biomed Mater Eng 2006;16:261-7. 
13. Kirshblum SC, Burns SP, Biering-Sorensen F, et al. International standards for neurological classification of spinal cord injury (revised 2011). J Spinal Cord Med 2011;34:535-46.

14. Ali Algadiem E, Aleisa AA, Alsubaie HI, Buhlaiqah NR, Algadeeb JB, Alsneini HA. Blood loss estimation using gauze visual analogue. Trauma Mon 2016;21:e34131.

15. Amhaz HH, Fox BD, Johnson KK, et al. Postlaminoplasty kyphotic deformity in the thoracic spine: case report and review of the literature. Pediatr Neurosurg 2009;45:151-4.

16. Park SB, Jahng TA, Kim CH, Chung CK. Thoracic and lumbar laminoplasty using a translaminar screw: morphometric study and technique. J Neurosurg Spine 2009;10:603-9.
17. Bertalanffy H, Mitani S, Otani M, Ichikizaki K, Toya S. Usefulness of hemilaminectomy for microsurgical management of intraspinal lesions. Keio J Med 1992;41:76-9.

18. Chiou SM, Eggert HR, Laborde G, Seeger W. Microsurgical unilateral approaches for spinal tumour surgery: eight years' experience in 256 primary operated patients. Acta Neurochir (Wien) 1989;100:127-33.

19. Ozawa H, Kokubun S, Aizawa T, Hoshikawa T, Kawahara C. Spinal dumbbell tumors: an analysis of a series of 118 cases. J Neurosurg Spine 2007;7:58793.

20. Naganawa T, Miyamoto K, Hosoe H, Suzuki N, Shimizu K. Hemilaminectomy for removal of extramedullary or extradural spinal cord tumors: medium to long-term clinical outcomes. Yonsei Med J 2011;52:121-9. 